\title{
Light Extraction Improvement of GaN LEDs using Nano-scale Top Transmission Gratings
}

\author{
Greg Chavoor ${ }^{\mathrm{a}}$, Xiaomin Jin ${ }^{\mathrm{a}}$, Ashton Ellaboudy ${ }^{\mathrm{a}}$, Xing-Xing Fu${ }^{\mathrm{b}}$, Xiang-Ning Kang ${ }^{\mathrm{b}}$, \\ Bei Zhang, and Guo-Yi Zhang ${ }^{\mathrm{b}}$ \\ ${ }^{a}$ Electrical Engineering Department, California Polytechnic State University, \\ San Luis Obispo, CA 93407, USA; \\ ${ }^{\mathrm{b}} \mathrm{School}$ of Physics and State Key Laboratory for Artificial Microstructures and \\ Mesoscopic Physics, Peking University, Beijing, 100871, China;
}

\begin{abstract}
In this paper, we use a Finite-Difference Time-Domain GaN LED model to study constant wave (CW) average power of extracted light. The structure simulated comprises of a $200 \mathrm{~nm}$-thick p-GaN substrate, 50nm-thick MQW, 400nm-thick n-GaN substrate, and a $200 \mathrm{~nm}$ n-GaN two-dimensional Photonic Crystal( $2 \mathrm{PhC})$ grating. We focus on optimizing three design parameters: grating period $(\mathrm{A})$, grating height (d), and fill factor (FF). In the primary set of simulations, we fix the fill factor at $50 \%$ and simulate ten different grating periods (100 to $1000 \mathrm{~nm}$ in steps of $100 \mathrm{~nm}$ ) and four different grating heights (50 to $200 \mathrm{~nm}$ in steps of $50 \mathrm{~nm}$ ), and calculate the average power output of the device. The results from these simulations show that for both conical and cylindrical gratings, the maxmium light extraction improvement occurs when $A=100 \mathrm{~nm}$. In the second set of simulations, we maintain a constant grating period $A=100 \mathrm{~nm}$ and sweep the fill factor from 25 to $75 \%$. The results of these simulations show that the fill factor affects clyindrical and conical gratings differently. As a whole, we see that the nano-structure grating mostly depends on period, but also depends on height and fill factor. The grating structure improves light extraction in some cases, but not all.
\end{abstract}

Keywords: Gallium Nitride, nano-scale, transmission grating, FDTD

\section{INTRODUCTION}

In the last 15 years, an immense amount of research has gone into developing $\mathrm{GaN}$ based light emitting diodes (LED) and laser diodes (LD). Shuji Nakamura paved the way for the GaN blue LED by developing new fabrication techniques and the first high power blue $\mathrm{LED}^{1,2}$. Today researchers continue to develop creative solutions that increase overall LED efficiency. LED performance can be improved in two ways: first by increasing electron hole pair recombination efficiency ${ }^{3}$, and second by increasing the light extraction efficiency ${ }^{4}$. Gallium Nitride's high refractive index reduces light emission via ghost mode formation in thick substrate and total internal reflection, which compete with the guided mode. The guided modes formation is resulted from total internal reflection and waveguide structure of LEDs. Ghost modes result from the waveguide-like properties of $\mathrm{GaN}$ and reduce the optical confinement factor of the device ${ }^{5}$, which greatly limits the LED's efficiency. However, in this paper, we consider thin film GaN LED, the ghost mode is not an issue here. Total internal reflection describes a phenomenon in which no light is transmitted at a boundary. Instead the light reflects at the boundary and stays within the original sending medium. The critical angle determines the angle above which light is totally internally reflected between two materials. A large difference in the refractive index of air and $\mathrm{GaN}$ results in a low critical angle and a significant amount of light trapped.

In this paper, we focus on light extraction efficiency. There are several ways to improve light extraction. Surface roughening methods and photonic crystal $(\mathrm{PhC})$ gratings implemented on the top layer of an LED provide more angles for light to escape and thus increase light extraction efficiency. ${ }^{6}$ Different patterns and

Eleventh International Conference on Solid State Lighting, edited by Matthew H. Kane, Christian Wetzel, Jian-Jang Huang, Proc. of SPIE Vol. 8123, 81231A - (c) 2011 SPIE - CCC code: 0277-786X/11/\$18 · doi: 10.1117/12.892665 
shapes of photonic crystal gratings can be formed by chemical wet etching, dry etching, and focused ion beam milling (FIBM). ${ }^{7}$ A few specific methods include: electron-beam lithography and nano-imprint lithography. ${ }^{8}$ In previous studies, simulations ${ }^{9-11}$ and experimental data ${ }^{12}$ show that the period, shape, and height of the grating all affect light extraction efficiency.

Fabricating and testing nano-structure gratings can be a difficult process that uses a large amount of resources. To reduce costs and development time, we simulate top layer nano-structure gratings and use the data as a fabrication guide tool. Previous work ${ }^{13}$ used Rigorous-Coupled Wave Analysis (RCWA) to show that a smaller period improves light extraction for the nano-structure device. For this paper, we use Finite Difference Time Domain (FDTD) to study improvements in light extraction efficiency. Section 2 of this paper quickly describes FDTD analysis and the device parameters of the LED model studied. Next, section 3 provides the simulation results for the conical and cylindrical gratings. Finally, section 4 concludes and discusses the findings from this research.

\section{SIMULATION SETUP}

\subsection{Simulation software}

In order to calculate improvements in light extraction, we use the Finite Difference Time Domain (FDTD) method. FDTD provides a solution to Maxwell's equations by using Yee's mesh, a system in which the E and $\mathrm{H}$ field components are solved based on the previous spatial $\mathrm{E}$ and $\mathrm{H}$ field components. ${ }^{14}$ This method requires the user to define the mesh size in the $\mathrm{x}, \mathrm{y}$ and $\mathrm{z}$ direction. Since 3-D simulations require significant time and memory, we perform all simulations in 2-D and only define the $\Delta \mathrm{x}$ grid and $\Delta \mathrm{y}$ grid. As a rule of thumb, the grid size should be at least smaller than one tenth the wavelength of light or the smallest dimension in the simulation profile. For the following simulations, the smallest dimension turned out to be smaller than one tenth the wavelength. Therefore, we chose $\Delta x=25 \mathrm{~nm}$ and $\Delta y=50 \mathrm{~nm}$.

\subsection{Simulation model}

Figure 1 displays an example of the thin-film LED simulated. The structure comprises of a $200 \mathrm{~nm} \mathrm{n}-\mathrm{GaN}$ $2 \mathrm{PhC}$ grating, $400 \mathrm{~nm} \mathrm{n}-\mathrm{GaN}$ substrate, $50 \mathrm{~nm} \mathrm{MQW}$, and $200 \mathrm{~nm} \mathrm{p}-\mathrm{GaN}$ substrate. Figure 1 also includes three essential FDTD simulation components: the FDTD launch, time monitor, and perfectly matched layer (PML). The launch serves as a source of light within the simulation, therefore we place it in the center of the multiple quantum wells (MQW) where most electron hole recombination occurs. We choose the light source to be constant wave (CW) at $460 \mathrm{~nm}$, assuming the LED to produce blue light. The light travels through the device until it reaches the time monitor. The time monitor takes a time average of power throughout the simulation and stores it in a separate data file. The PML surrounds the simulation window and allows waves to exit without producing any unwanted reflections. Generally, the PML should be ten or more grid points thick in each direction $(\mathrm{x}, \mathrm{y}, \mathrm{z})$, and in this case $(\mathrm{x}, \mathrm{y})$. 


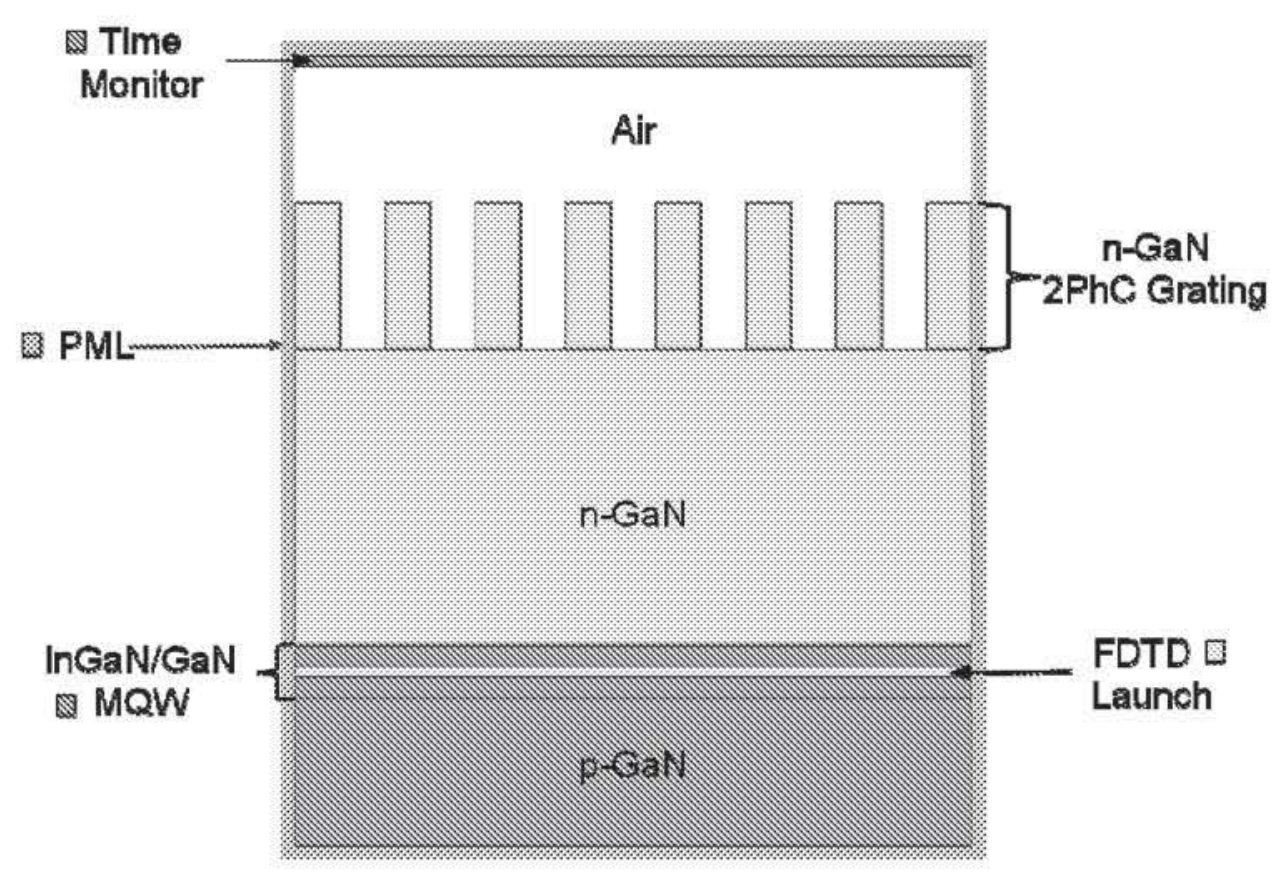

Figure 1. Thin film LED simulation model.

Table 1 provides information on the material used, such as the refractive index and thickness. We focus on optimizing three design parameters: grating period (A), grating height (d), , and fill factor (FF). The grating period $\mathrm{A}$ is the distance between the centers of each grating cell and the fill factor (FF) represents the percentage of $\mathrm{GaN}$ within one period.

Figure 2 shows a closeup of the grating structure with $\mathrm{FF}=75 \%$. In one period $75 \%$ of the grating is filled with $\mathrm{GaN}$ and $25 \%$ is filled by air. We use a refractive index of 2.5 for all $\mathrm{n}$ and $\mathrm{p}$ type GaN substrates except the InGaN/GaN MQW which has an index of refraction equal to 2.6 It is also important to notice that each material has a constant thickness except the $2 \mathrm{PhC}$ grating structure which varies from 50 to $200 \mathrm{~nm}$ in steps of $50 \mathrm{~nm}$.

Table 1. List of LED materials and their respective refractive index and thickness.

\begin{tabular}{|c|c|c|}
\hline Material & Refractive Index & Thinness (nn) \\
\hline Air & 1 & N/A \\
\hline n-GaN 2PhC Grating & 2.5 & $50-200$ \\
\hline n-GaN & 2.5 & 400 \\
\hline InGaN/GaN MQW & 2.6 & 50 \\
\hline p-GaN & 2.5 & 200 \\
\hline
\end{tabular}




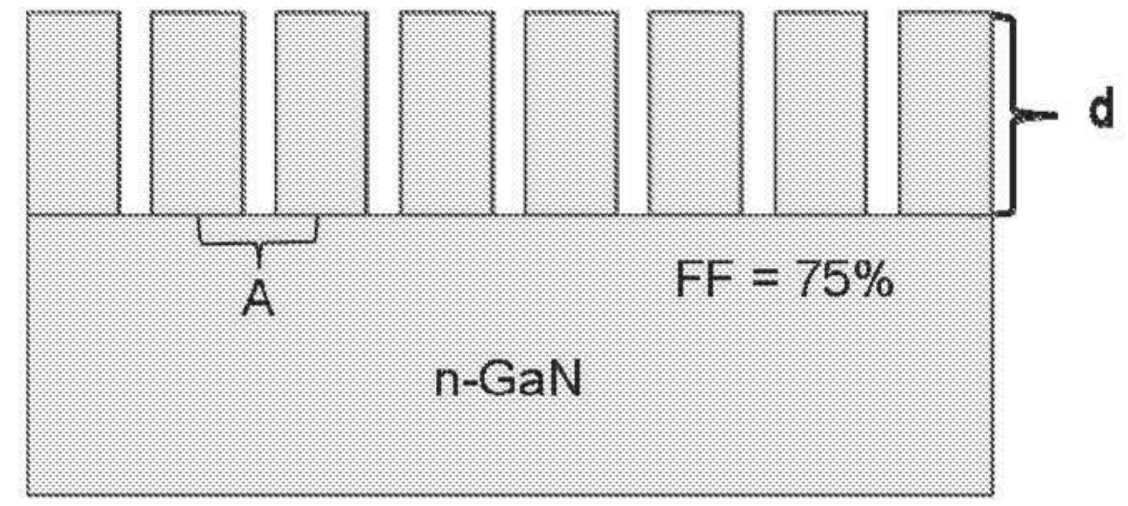

Figure 2. Close up of the nano-scale grating with fill factor $=75 \%$.

\section{SIMULATION RESULTS}

\subsection{Cylindrical and conical gratings}

In order to optimize device light extraction, we compare average power data from three basic models: the first has no grating, the second has a cylindrical shaped grating, and the third has a conical shaped grating. Since we simulated in 2-D, the cylindrical grating appears as a rectangular and the conical grating appears to be a triangular. The first case, with no grating, serves as a base value to compare other results against. Any improvements in light extraction are based off the results from this model. Since the grating period (A) and fill factor (FF) cannot be changed for this model, we simply change the height (d) from 50 to $200 \mathrm{~nm}$ in steps of $50 \mathrm{~nm}$. Therefore the total n-GaN substrate height changes from $600 \mathrm{~nm}$ to $450 \mathrm{~nm}$.

Next, for the cylindrical and conical models, we set the fill factor to $50 \%$ and sweep the grating period from $100 \mathrm{~nm}$ to $1000 \mathrm{~nm}$ in steps of $100 \mathrm{~nm}$. For each period, we also change the grating height from $50 \mathrm{~nm}$ to $200 \mathrm{~nm}$ in steps of $50 \mathrm{~nm}$. The results for the cylindrical model (displayed in Figure 3) show that light extraction improves only for a grating period of $100 \mathrm{~nm}$. Unexpectedly, in all other cases the grating reduces light extraction compared to the non-grating model. In the case of the cylindrical model, maximum average power occurs when the grating period $A=100 \mathrm{~nm}$ and grating height $\mathrm{d}=50 \mathrm{~nm}$. In this case, light extraction increases by $10.8 \%$ compared to the non-grating model with $\mathrm{d}=200 \mathrm{~nm}$. The general trend shows that as grating period increases, light extraction decreases. However, above $400 \mathrm{~nm}$ light extraction begins to improve but fails to exceed the non-grating performance. The trend for grating height effects is not as well defined as the grating period. In some cases, such as $\mathrm{A}=300 \mathrm{~nm}$, a larger grating height improves efficiency and in other cases, such as $\mathrm{A}=100 \mathrm{~nm}$, the smallest grating height improves efficiency. However, for grating periods $\mathrm{A}>300 \mathrm{~nm}$ it seems as though an increase in grating height generally decreases average power. 

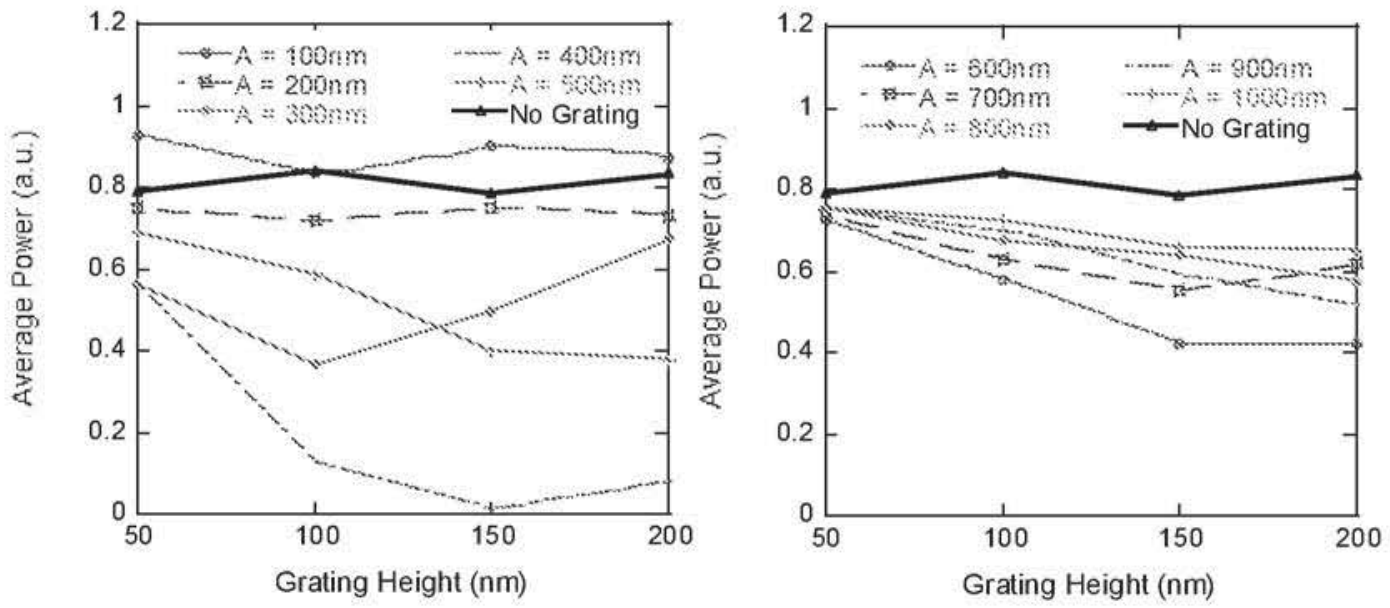

Figure 3. Average power plotted versus grating height for cylindrical grating.

The results for the conical model, displayed in Figure 4, show that light extraction improves for grating period $\mathrm{A}=100 \mathrm{~nm}$ and in some cases $\mathrm{A}=200 \mathrm{~nm}$. All other grating periods result in decreased light extraction relative to the non-grating model. Unlike the cylindrical model, maximum average power occurs when $A=100 \mathrm{~nm}$ and $\mathrm{d}=100 \mathrm{~nm}$. In this case light extraction increases by $16.8 \%$ relative to the nongrating model with height $\mathrm{d}=200 \mathrm{~nm}$. Relative to the cylindrical model maximum, the conical model increases light extraction by $5.4 \%$. The general trend shows similarities to the cylindrical model. An increase in grating period results in a decrease in light extraction. When $\mathrm{A}>400 \mathrm{~nm}$ light extraction begins to improve, but never reaches non-grating performance. For cases where $\mathrm{A}>300 \mathrm{~nm}$, an increase in grating height seems to decrease overall performance.
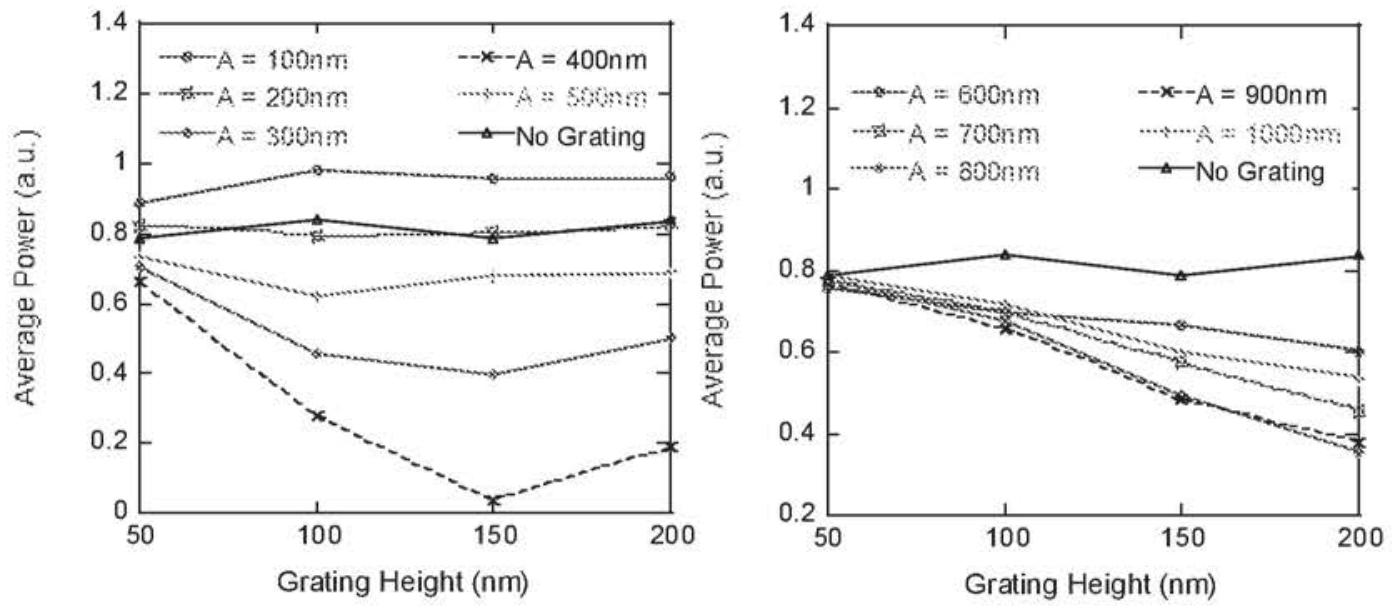

Figure 4. Average power plotted versus grating height for conical grating. 


\subsection{Changing the fill factor}

In the final set of simulations, we set the grating period to $100 \mathrm{~nm}$ and alter both the grating height and fill factor. We maintain a grating period of $100 \mathrm{~nm}$ because the most improvement in both devices occurs at this period as seen in Figure 3 and Figure 4 . We alter the grating height from $50 \mathrm{~nm}$ to $200 \mathrm{~nm}$ in steps of $50 \mathrm{~nm}$ and change the fill factor to the following five values: $25 \%, 33 \%, 50 \%, 66 \%$, and $75 \%$. The results for the cylindrical grating in Figure 5 show a $17.8 \%$ increase in light extraction for grating height $d=200 \mathrm{~nm}$ and $\mathrm{FF}=33 \%$ compared to the non-grating case when $\mathrm{d}=200 \mathrm{~nm}$. It also shows a $6.3 \%$ increase compared to the previous cylindrical maximum value. The results for the conical grating in Figure 5 show a $17.8 \%$ increase in average power for grating height $\mathrm{d}=200 \mathrm{~nm}$ and $\mathrm{FF}=66 \%$ relative to the non-grating case where $d=200 \mathrm{~nm}$. In general, increasing or decreasing the fill factor from $50 \%$ results in an increase in light extraction.

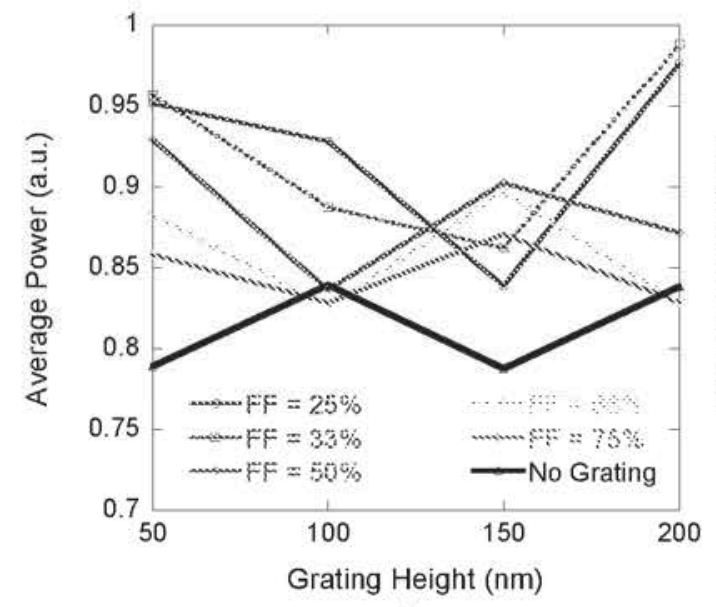

(a)

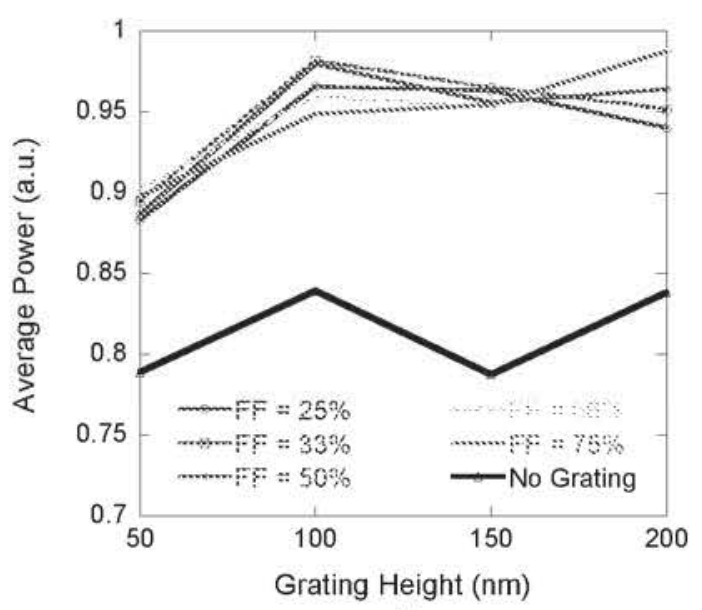

(b)

Figure 5. (a) Cylindrical grating average power for several fill factors; (b) conical grating average power for several fill factors.

Table 2 serves to clarify and summarize the results of this study. It lists the grating type, grating period (A), grating height (d), fill factor (FF), average power, and the increase in light extraction relative to the nongrating case where $d=200 \mathrm{~nm}$. The average power column illustrates that different non-grating cases have different light extraction efficiencies; we used the $d=200 \mathrm{~nm}$ case to compare all other data against. The table also highlights the two structures with the highest light extraction by using boldface font. 
Table 2. Summary of maximum light extraction data.

\begin{tabular}{|c|c|c|c|c|c|}
\hline Grating Type & $\mathrm{A}(\mathrm{nm})$ & $d(n m)$ & $\mathrm{FF}(\%)$ & $\begin{array}{l}\text { Average } \\
\text { Power (a.u.) }\end{array}$ & $\begin{array}{l}\text { Relative } \\
\text { Increase }(\%) \text { ) }\end{array}$ \\
\hline \multirow[t]{2}{*}{ Cylindrical } & 100 & 50 & 50 & 0.929 & 10.8 \\
\hline & 100 & 200 & 33 & 0.988 & 17.8 \\
\hline \multirow[t]{2}{*}{ Conical } & 100 & 100 & 50 & 0.979 & 16.8 \\
\hline & 100 & 200 & 66 & 0.988 & 17.8 \\
\hline \multirow[t]{4}{*}{ Non-Grating } & $\mathrm{n} / \mathrm{a}$ & 50 & $\mathrm{n} / \mathrm{a}$ & 0.78913 & 0 \\
\hline & $\mathrm{n} / \mathrm{a}$ & 100 & $\mathrm{n} / \mathrm{a}$ & 0.83955 & 0 \\
\hline & $\mathrm{n} / \mathrm{a}$ & 150 & $\mathrm{n} / \mathrm{a}$ & 0.78778 & 0 \\
\hline & $\mathrm{n} / \mathrm{a}$ & 200 & $\mathrm{n} / \mathrm{a}$ & 0.83856 & 0 \\
\hline
\end{tabular}

\section{CONCLUSION}

FDTD analysis shows that a nano-structure top grating does improve light extraction efficiency only for small grating period. Most grating periods larger than $100 \mathrm{~nm}$ resulted in lower efficiency. In the case where fill factor is set to $50 \%$, the conical grating showed a $16.7 \%$ increase in average power compared to the non-grating model and a 5.4\% compared to the cylindrical model. Changing the fill factor showed that, in general, the conical model outperforms the cylindrical model. However, the clyindrical and conical models obtain the same maximum average power. The cylindrical model's maximum average power occurred for $\mathrm{A}=100 \mathrm{~nm}, \mathrm{~d}=200 \mathrm{~nm}$, and $\mathrm{FF}=33 \%$. The conical model's maximum average power occurred for $\mathrm{A}=100 \mathrm{~nm}, \mathrm{~d}=200 \mathrm{~nm}$, and $\mathrm{FF}=66 \%$. These results provide an outline to design thin film $\mathrm{GaN}$ based LED's with a nano-structure grating.

\section{ACKNOWLEDGEMENTS}

This project is supported by 1) NSF Grant IRES Award \#1029135 from year 2010 to 2013 and 2) Chinese National Key Research Lab Collaboration Grant 2010-2011, Peking University in China.

\section{REFERENCES}

[1] Nakamura, S., Senoh, M., and Mukai, T., "High-power InGaN/GaN double-heterostructure violet light emitting diodes," Appl. Phys. Lett., 62(19), 2390-2392 (1993).

[2] Nakamura, S., Harada, Y., and Seno, M., "Novel metalorganic chemical vapor deposition system for GaN growth," Appl. Phys. Lett. 58(18), 2021-2023 (1991).

[3] Gardner, N. F., and Chui, H.C., "1.4x efficiency improvement in transparent-substrate $\left(\mathrm{Al}_{\mathrm{x}} \mathrm{Ga}_{1}\right.$. x $)_{0.5} \mathrm{In}_{0.5} \mathrm{P}$ light-emitting diodes with thin $(\leq 2000 \AA)$ active regions," Appl. Phys. Lett., 74(15), 22302232 (1999). 
[4] Dai, T. and Zhang, B., "Light Extraction Improvement from GaN-Based Light-Emitting Diodes with Nano-Patterned Surface Using Anodic Aluminum Oxide Template," IEEE Photonics Technology Letters, 20(23), 1974-1976 (2008).

[5] Eliseev, P.G., Smolyakov, G.A., and Osinski, M., "Ghost Modes and Resonant Effects in AlGaNInGaN—GaN Lasers," IEEE Journal of Selected Topics in Quantum Electronics, 5(3), 771-779 (1999).

[6] Lin, C. and Chen, C., "Light Extraction Enhancement of a GaN-Based Light-Emitting Diode Through Grating-Patterned Photoelectrochemical Surface Etching with Phase Mask Interferometry," IEEE Photonics Technology Letters, 22(9), 640-642 (2010).

[7] Dai, T., and Kang, X., "Study and formation of 2D microstructures of sapphire by focused ion beam milling," Microelectronic Engineering, 85(3), 640-645 (2008).

[8] Kim, S., and Lee, K., "Fabrication of photonic crystal structures on light emitting diodes by nanoimprint lithography," Nanotechnology, 18(5), 1150(2007).

[9] Trieu, S., and Jin, X., "Study of Top and Bottom Photonic Gratings on GaN LED with Error Grating Models," IEEE Journal of Quantum Electronics, 46 ( 10 ) , 896-901 (2010).

[10] Bao, K. and Kang, X.N., "Improvement of Light Extraction from Patterned Polymer Encapsulated GaN-Based Flip-Chip Light-Emitting Diodes by Imprinting," IEEE Photonics Technology Letters, 19(22), 1840-1842 (2007).

[11]Ryu, H. and Shim, J., "Structural Parameter Dependence of Light Extraction Efficiency in Photonic Crystal InGaN Vertical Light-Emitting Diode Structures," IEEE Journal of Quantum Electronics, 46(5), 714-720 (2010).

[12] Lin, Y. Z. and Kong, F.M., "FDTD Study on the Improvement of Optical Transmission through Metallic Periodic Nano Structure," Proc. OptoElectronics and Communications Conference, 1-2 (2009).

[13] Jin, X. and Trieu, S., "Design Simulation of Top ITO Gratings to Improve Light Transmission for Gallium Nitride LEDs," Proc. Information Technology: New Generations, 1-4 (2009).

[14] Yee, K., "Numerical solution of initial boundary value problems involving Maxwell's equations in isotropic media," IEEE Trans. Antennas Propa.., 14(3), 302-307(1966). 